\title{
Attenuating the Post-Stroke Epilepsy Using the Si-Miao-Yong-An Decoction for Treatment
}

\author{
Shisheng Jiang ${ }^{1}$, Mukut Sharma ${ }^{2}$, Yuming Han ${ }^{1}$, Hongmei Wang ${ }^{1}$, Feizhou He ${ }^{3}$, \\ Shuangteng $\mathrm{He}^{1,3,4}$, * \\ ${ }^{1}$ Institute of Clinical Research, Hunan Academy of Traditional Chinese Medicine, Changsha, China \\ ${ }^{2}$ Renal Research Laboratory, and the Midwest Veterans' Biomedical Research Foundation (MVBRF), VA Medical Center, Kansas City, USA \\ ${ }^{3}$ Shuangteng Rehabilitation Hospital of TCM, Loudi, China \\ ${ }^{4}$ Stroke Research Laboratory, VA Medical Center, Kansas City, USA
}

Email address:

shisheng.jiang@yahoo.com (Shisheng Jiang), Mukut.Sharma@va.gov (Mukut Sharma), hanyum@126.com (Yumin Han), 178229718@qq.com (Hongmei Wang), feizhouh@gmail.com (Feizhou He), shuangteng@aliyun.com (Shuangteng He)

${ }^{*}$ Corresponding author

\section{To cite this article:}

Shisheng Jiang, Mukut Sharma, Yuming Han, Hongmei Wang, Feizhou He, Shuangteng He. Attenuating the Post-Stroke Epilepsy Using the Si-Miao-Yong-An Decoction for Treatment. International Journal of Chinese Medicine. Vol. 4, No. 3, 2020, pp. 54-60.

doi: $10.11648 /$ j.ijcm.20200403.12

Received: July 7, 2020; Accepted: July 27, 2020; Published: August 20, 2020

\begin{abstract}
Objective: To observe the clinical efficacy of Si-Miao-Yong-An decoction (SMYAD) in the treatment of post-stroke epilepsy. Methods: 112 patients with post-stroke epilepsy were randomly assigned to either the control or the treatment group (56 subjects in each group). Patients in the control group received oral levetiracetam (LEV) combined with other conventional Western medicine treatment (s), while the treatment group received SMYAD only. Outcome measures were the duration for seizure remission, remission rates at one year, changes in electroencephalogram (EEG), scores of National Institutes of Health Stroke Scale (NIHSS), and adverse events in the two groups. Results: The numbers of patients who completed treatment were 41 and 55 in the LEV group and SMYAD group, respectively $(P<0.01)$. After 12 months of treatment, epilepsy was controlled in 35 patients in LEV group and 47 in SMYAD group $(P=0.990)$. Seizure remission was achieved in 15.1 days in LEV group and 8.7 days in SMYAD group $(P<0.01)$. Relapse of epilepsy was observed in 19 cases in the LEV group and 11 in SMYAD group $(P<0.01)$. Abnormal EEG patterns persisted in 32 patients in LEV group and 29 patients in SMYAD group $(P<0.01)$. The NIHSS score decreased from 12.2 to 9.4 in LEV group, and from 12.5 to 5.8 in SMYAD group $(P<0.01)$. Adverse effects of treatment were observed in 28 cases in LEV group and 6 patients in SMYAD group $(P<0.01)$. The most common adverse events reported were rash, fatigue, somnolence, headache, and dizziness in LEV group, and diarrhea in SMYAD group. Adverse effects caused withdrawal of 8 patients from LEV group but none from SMYAD group $(P<0.01)$. Conclusion: SMYAD is an effective and well tolerated remedy for post-stroke epilepsy. Further large scale randomized controlled trials are needed to establish the efficacy and tolerability.
\end{abstract}

Keywords: Post-Stroke Epilepsy, Traditional Chinese Medicine Treatment, Si-Miao-Yong-An Decoction

\section{Introduction}

Cerebrovascular disease including stroke is the leading cause of symptomatic epilepsy in elderly accounting for $11 \%$ of new onset epilepsy, $22 \%$ of incident status epilepticus, and $30 \%$ of acute symptomatic seizures [1-4]. Numerous clinical studies have shown higher incidence of post-stroke epilepsy in hemorrhagic stroke as compared to ischemic stroke with cortical hemorrhage being an independent and most important predictor of epilepsy [5-9]. Intracranial hemorrhage leads to iron deposition in brain tissue and may become an important factor resulting in epilepsy. Experimental studies demonstrated that injection of iron into brain tissue induced release of glutamic and aspartic acids into extracellular space and caused spontaneous epileptiform of electroencephalogram (EEG) and chronic spontaneous recurrent seizures or epilepsy 
in animal models [10-13]. Even though ischemic stroke occurs secondary to vascular occlusive disease with no hemorrhage, it is associated with excessive iron accumulation in the infarct area due to multiple mechanisms including infiltration by peripheral blood monocytes and macrophages and increased heme synthesis by neuroglial cells [14-16].

Free iron generates reactive oxygen species (ROS) in aqueous solutions or biologic fluids and induces inflammation by activating nuclear factor kappa B (NF- $\mathrm{B})$ and toll-like receptor 4 (TLR-4) [17-19]. Oxidative injury and neuroinflammation are being increasingly recognized as an important factor in epileptogenesis. Pro-inflammatory cytokines such as interleukin-1 $\beta$ (IL-1 $\beta$ ) can promote neuronal excitability and induce seizures [20, 21]. Considering the plethora of evidence supporting a strong association between iron and development of post-stroke epilepsy, attenuating oxidant stress and inflammatory response by removing excessive iron from the brain may reduce the occurrence of post-stroke epilepsy. Current mainstay of post-stroke epilepsy treatment is with anti-epileptic drugs (AED). However, AED's only provide symptomatic therapy with no effect on epileptogenesis in stroke patients [22-28]. Levetiracetam (LEV) is one of the widely used and well tolerated AED but has no antiepileptogenic effect and no effect on removing iron in the stroke bed [27]. Due to the neurotoxicity of free iron, deferoxamine (DFO), an iron chelator, was used to chelate and clear free iron from the brain for patients with hemorrhagic stroke [29]. However, effect of DFO on the incidence of post-stroke seizures or post-stroke epilepsy are unclear.

We have used Si-Miao-Yong-An decoction (SMYAD) to treat patients with stroke for more than 20 years and found it could improve the clinical recovery of neurological functions and prevent recurrence of stroke [30-32]. We recently observed that administration of SMYAD resulted in seizure-control in 35 patients with post-stroke epilepsy [33]. SMYAD is composed of four herbs, also named as four powerful herbs decoction (FPHD). SMYAD has been used to treat arteritis and arterial blockage in extremities for more than one hundred years in traditional Chinese medicine (TCM) [30] It is now widely used in the treatment of ischemic heart disease resulting from coronary atherosclerosis and ischemic stroke due to atheromatous plaque formation in carotid artery [34-36]. Recent experimental studies found it can alleviate oxidative stress and inflammatory response by inhibiting $\mathrm{NF}-\kappa \mathrm{B}$ expression in endothelium of artery, and by decreasing the levels of malondialdehyde (MDA), interleukin-1 $\beta$ (IL-1 $\beta$ ), tumor necrosis factor- $\alpha$ (TNF- $\alpha)$ and monocyte chemoattractant protein-1 (MCP-1) in serum in the animal model of atherosclerosis [37, 38]. More recently, we found that SMYAD could decrease iron content in rat the brain after ICH in animal models [39]. Based on these findings, we designed this study to evaluate the effects of SMYAD on reducing post-stroke epilepsy in patients compared to LEV controls.

\section{Subjects and Methods}

\subsection{Study Population}

From January 2012 to October 2019, we recruited 112 patients with post-stroke epilepsy at the Affiliated Hospital of Hunan Academy of traditional Chinese Medicine (TCM) and the Shuangteng Rehabilitation Hospital of TCM in Hunan Province, China. The diagnosis of stroke and its sub-types, i.e., intracerebral hemorrhage (ICH), subarachnoid hemorrhage (SAH), or cerebral infarction (CBI), were based on the clinical signs and computerized tomography (CT) scan or magnetic resonance imaging (MRI) of the head according to the guidelines from the American Heart Association /American Stroke Association [40, 41]. Post-stroke epilepsy was diagnosed from medical records written by medical staff at the study hospitals or from reliable descriptions provided by family members who witnessed the occurrence of seizures. In this study, post-stroke seizures were defined as single or multiple convulsive episodes after stroke, and distinguished as early (occurring within 14 days after stroke onset) or late (occurring 14 days after stroke onset) seizures according to the literature $[4,26]$, and classified as focal generalized seizures according to the criteria of Commission on Classification and Terminology of the International League Against Epilepsy [42]. Post stroke epilepsy was defined as recurrent seizures following stroke. All patients enrolled in this study were diagnosed as post-stroke epilepsy with confirmed records of recurrent seizures after stroke onset. Patients with history of epilepsy before stroke were excluded from the study.

The research protocol was approved by the Institutional Ethics Committees of Hunan Academy of TCM and Shuangteng Rehabilitation Hospital of TCM. The patients enrolled in this study or one of their family members, in case of memory impairment of the objects, were provided with and signed the informed consent. The patients or the family member were made aware of the treatment plans.

\subsection{Study Design}

Patients were randomly and equally divided into SMYAD or LEV groups following the randomization scheme (see Table 1 for details). Treatment was started on the first day when the patient was enrolled in the study and continued for 12 months. No other AEDs were allowed during the study period. If any other AED was added, the patient was counted as non-responder and was dropped from the study.

\subsubsection{LEV Treatment}

LEV (Shenghuaxi Pharmaco Co. Ltd., Chongqing, China) was given orally at starting dose of $500 \mathrm{mg}$ twice daily (1000 $\mathrm{mg} /$ day) for the first 20 days. If no effective signs were seen, the dose was increased to $1000 \mathrm{mg}$ twice daily $(2000 \mathrm{mg} /$ day $)$ from day 21 and continued to the end of observation. A gradual dose reduction of $500 \mathrm{mg}$ per week was applied after the end of one-year clinical observation. LEV was replaced by other AEDs for patients whose epilepsy was not controlled during the study period. 


\subsubsection{SMYAD Treatment}

The original formulation of SMYAD contains Jin-yin-hua (Honeysuckle flower) 30 grams (g), Xuan-shen (Scrophularia root) 30g, Dang-gui (Chinese angelica root) $15 \mathrm{~g}$, and Gan-cao (Licorice root) $10 \mathrm{~g}$. We added Gou-qi-zi (Wolfberry fruit) $15 \mathrm{~g}$, Bai-mao-gen (Cogongrass rhizome) 15g, Che-qian-zi (Plantain seed) 10g, Tian-ma (Gastrodia tuber) $10 \mathrm{~g}$, and Ju-hua (Chrysanthemum flower) $10 \mathrm{~g}$. This is a dosage for 2 days for an adult patient. Changes to this formulation of the decoction are made according to symptoms by adding or removing one or more herbs. For example, Yuan-zhi (Polygala root) 5g and Suan-zao-ren (Wild jujube seed) $15 \mathrm{~g}$ were added for patients with cognitive impairment; Yuan-zhi (Polygala root) 5g and Shi-chang-pu (Grassleaved sweetflag rhizome) 10g were added for those with aphasia; Ban-xia (Pinellia tuber) 6g, Zhu-ru (Bamboo shavings) 15g, Chen-pi (Tangerine peel) $10 \mathrm{~g}$, and Dan-nan-xing (Arisaema cum bile) $5 \mathrm{~g}$ were added for those with phlegm in the body; Ren-shen (Ginseng) 10 grams and Huang-qi (Astragalus root) 20g were added for those with Qi deficiency; and Ge-gen (Pueraria root) 10g, Fu-ling (Poria) 10g, and Bai-zhu (White Atractylodes rhizome) $6 \mathrm{~g}$ were added and Xuan-shen (Scrophularia root) was removed for patients with diarrhea. All these herbs were boiled in $800 \mathrm{ml}$ tap water for about 40 minutes to get about $400 \mathrm{ml}$ of decoction at the first time, and the cooked herbs was boiled again in $600 \mathrm{ml}$ water to get the second decoction of approximately $400 \mathrm{ml}$. The first and second decoctions were mixed, and patients took it twice daily (in the morning and evening) for 2 days with $200 \mathrm{ml}$ decoction each time. Thus, each preparation (dose) of decoction lasted for 2 days. Fifteen such doses were prepared each month, the total period of treatment being 12 months.

Both LEV and raw herbs in SMYAD were provided to patients by the pharmacies in Hunan Academy of TCM or in Shuangteng Rehabilitation Hospital of TCM according to assigned randomized numbers.

\subsection{EEG Assessment}

All patients were examined using a digital EEG System (NCC Medical Co., Ltd, Shanghai, China) at the beginning and end of the study for epileptiform discharges. Presence of epileptiform discharges were considered as an important ancillary data in the diagnosis and management of epilepsy but not as a diagnostic criterion with epilepsy being a clinical diagnosis.

\subsection{Data Collection}

Demographics, general physical exam, neurological exam, epilepsy history, seizure frequency, neuroimaging, EEG and laboratory data were collected and recorded at the time of enrollment and at the end of the study. Data were stored at the Medical Records Center at the Hunan Academy of TCM or at Shuangteng Rehabilitation Hospital of TCM and are available upon request. Once the treatment was started, data on seizures frequency and neurological functions at the end of each month were collected by a physician and a duty nurse from the research group. Seizure frequency and status were recorded using "Seizure Diaries" by patients or caregivers with date, time, and duration of seizures. Disability from stroke was assessed with the National Institute of Health Stroke Scale (NIHSS).

\subsection{Criteria for Clinical Efficacy of the Treatment}

\subsubsection{The Efficacy of Treatment}

The clinical efficacy of the treatment for epilepsy was set as controlled, uncontrolled, and relapse according to published literature [22-27]. Controlled: no more seizures occurred from 8 weeks after the treatment to the end of observation. Uncontrolled: one or more seizures still occurred after 8 weeks of treatment. Relapse: epilepsy was controlled during the 12-month treatment, but it reoccurred during the following 6-month observation after treatment.

\subsubsection{Adverse Events}

Occurrence of unexpected symptoms during the treatment such as rash, diarrhea, headache, that could not be ascribed to any other causes, was defined as adverse events.

\subsubsection{EEG Changes}

Disappearance of epileptic waves from the EEG after treatment was regarded as improved EEG or normal EEG.

\subsection{Statistical Analysis}

Data were summarized as mean $\pm \mathrm{SD}$, and comparisons between the two groups were made using the unpaired $t$-test. Rank sum test was used for gradational data. Chi-square $\left(\chi^{2}\right)$ test was used for counting data. The differences between the groups were considered statistically significant at $P<0.05$.

\section{Results}

\subsection{General Information on Patients}

Total 112 patients were randomly divided to LEV and SMYAD groups (56 in each group). The average age was 64.5 year in LEV group (the youngest was 27 years, and the oldest was 86), and was 64.6 in SMYAD group (the youngest was 26 years, and the oldest was 87). There were 32 male and 24 female subjects in LEV group, and 31 male and 25 female subjects in SMYAD group. The course length of epilepsy (from the recurrence of seizures after stroke to the enrollment of this study) was 5.5 months (1-20-month range) in LEV group and 5.3 months (1-17 month) in SMYAD group. The severity of stroke in all patients was assessed using the NIH Stroke Scale (NIHSS). These scores were between 5 and 21 for the mildest and most severe stroke experienced by subjects. The age, gender, course length of epilepsy, subtypes of stroke, kinds of epilepsy, NIHSS scores, and abnormal ECG of patients were shown in Table 1, and no differences were found between the two groups (All $P>0.05)$. 
Table 1. The age, gender, subtype of diseases, NIHSS, EEG, and treatment completion of patients.

\begin{tabular}{llll}
\hline Group & LEV & SMYAD & P Value \\
\hline Cases & 56 & 56 & \\
Age (Year) & $64.5 \pm 11.9$ & $64.6 \pm 13.6^{\mathrm{a}}$ & 0.971 \\
Gender (M/F) & $32 / 24$ & $31 / 25^{\mathrm{a}}$ & 0.849 \\
The course length of epilepsy (Month) & $5.5 \pm 1.6$ & $5.3 \pm 1.7^{\mathrm{a}}$ & 0.877 \\
Subtype of Stroke: ICH & 34 & $32^{\mathrm{a}}$ & 0.701 \\
SAH & 5 & $5^{\mathrm{a}}$ & 1.000 \\
CBI & 17 & $19^{\mathrm{a}}$ & 0.686 \\
Type of epilepsy: Focal & 31 & $30^{\mathrm{a}}$ & 0.850 \\
Generalized & 25 & $26^{\mathrm{a}}$ & 0.850 \\
NIHSS (before treatment) & $12.2 \pm 3.4$ & $12.5 \pm 3.5^{\mathrm{a}}$ & 0.660 \\
Abnormal EEG & 50 & $49^{\mathrm{a}}$ & 0.768 \\
Cases Completing Treatment & 41 & $55^{\mathrm{b}}$ & 0.000 \\
Withdrawal due to Economic Reason & 4 & $0^{\mathrm{b}}$ & 0.042 \\
Withdrawal due to Adverse effects & 8 & $0^{\mathrm{b}}$ & 0.006 \\
Death & 3 & $1^{\mathrm{a}}$ & 0.309 \\
Suicide & 2 & $0^{\mathrm{a}}$ & 0.154 \\
Sever Pneumonia & 1 & $1^{\mathrm{a}}$ & 1.000 \\
\hline
\end{tabular}

Note: a: $P>0.05$, b: $P<0.05$ ( $t$ or $\chi^{2}$ tests, compared with LEV group).

\subsection{The number of Patients Completing Treatment}

Of the patients enrolled (56 in each group), 41 patients from LEV group and 55 cases from SMYAD group completed the therapeutic course $(P<0.001)$. As shown in Table 1 , some subjects withdrew from the treatment due to a variety of reasons. Economic reasons- 5 individuals withdrew from LEV group due to higher cost of LEV treatment compared to none from SMYAD group $(P<0.05)$; Adverse events during treatment- 8 patients from LEV group discontinued treatments due to side effects compared to none in SMYAD $(P<0.05)$; Death- 3 patients in LEV group (2 suicides, 1 lung infection) and 1 patient in SMYAD group (lung infection) died during the study period $(P>$ 0.05). These data showed that more patients in SMYAD group completed the treatment than in LEV group indicating that SMYAD was better tolerated than LEV.

\subsection{Epilepsy Control}

Table 2 shows that 35 of 41 cases (35/41) in LEV group and 47 of 55 cases $(47 / 55)$ in SMYAD group got their epilepsy controlled during the one-year treatment period. Thus, control of epilepsy was achieved in $85.36 \%$ of cases in LEV group, and $85.45 \%$ in SMYAD group $(P>0.05)$ indicating a comparable effect of the two treatment plans. The time for onset of epilepsy control by treatment was 8.7 days in SMYAD group and 15.1 days in LEV group $(P<0.01)$. During the following 6-month observation post-treatment, relapse of epilepsy occurred in 19 (19/38) patients in LEV group and 11 $(11 / 51)$ in SMYAD group. These findings indicate a lower rate of relapse in SMYAD group compared to LEV group $(21.3 \%$ versus $50.0 \%, P<0.01)$. These data showed beneficial effects of SMYAD on controlling post-stroke epilepsy and reducing its relapse.

Table 2. The effects of SMYAD on controlling seizures and improving EEG and NIHSS score.

\begin{tabular}{llll}
\hline Group & LEV & SMYAD & P Value \\
\hline Course Completed (n) & 41 & $55^{\mathrm{b}}$ & 0.000 \\
Epilepsy Controlled (n) & 35 & $47^{\mathrm{a}}$ & 0.990 \\
Onset Time of Seizures Controlled (day)* & $15.1 \pm 5.5$ & $8.7 \pm 4.1^{\mathrm{b}}$ & 0.000 \\
EEG (n): Before Treatment (Abnormal/Total) & $50 / 56$ & $49 / 56^{\mathrm{a}}$ & $29 / 55^{\mathrm{b}}$ \\
After Treatment (Abnormal/Completed) & $32 / 41$ & $12.5 \pm 3.5^{\mathrm{a}}$ & 0.768 \\
NIHSS Score: Before Treatment & $12.2 \pm 3.4$ & $5.8 \pm 3.5^{\mathrm{b}}$ & 0.011 \\
After Treatment & $9.4 \pm 3.7$ & $11^{\mathrm{b}}$ & 0.660 \\
Seizures Relapse (n) & 19 & 0.000 \\
\hline
\end{tabular}

Note: a: $P>0.05$, b: $P<0.05$ ( $t$ test or $\chi^{2}$ test, compared with LEV group).

* Only the data from cases with seizures controlled were analyzed.

\subsection{Improvement in EEG Patterns}

As shown in Table 2, abnormal EEG patterns persisted in 32 patients in LEV group from 41 completed cases, and in 29 patients in SMYAD group from 55 completed cases. These data indicate normalization of EEG in more patients from SMYAD group compared to LEV group $(P<0.05)$.

\subsection{Improvement in NIHSS Score}

As shown in Table 2, the NIHSS score in SMYAD group decreased from 12.5 to 5.8, while that in LEV group decreased from 12.2 to 9.4 after one-year treatment. The difference between the groups was significant $(P<0.01)$. These results support our previous findings that SMYAD is beneficial for the recovery of neurological function in stroke patients [29-31].

\subsection{Adverse Events}

As shown in Tables 1 and 3, 28 patients in LEV group and 6 patients in SMYAD group suffered from advents events of treatments $(P<0.05)$. The number of subjects who withdrew from the study due to severe symptoms was 8 and none in LEV and SMYAD groups, respectively $(P<0.01)$. The symptoms of advents events mostly were rash, fatigue, somnolence, headache, dizziness in LEV group, and diarrhea in SMYAD group (Table 3). Most of these symptoms could be ameliorated with suitable medications. For example, the headache observed in LEV group could be relieved by ibuprofen, and diarrhea in SMYAD group was easily moderated by excluding Xuan-shen (Scrophularia root) and adding Ge-gen (Pueraria root), Fu-ling (Poria), and Bai-zhu (White Atractylodes rhizome). Some stroke patients experienced constipation and SMYAD treatment improved 
their bowel movement (data not shown). However, some adverse symptoms of LEV such as rash or dizziness of could not be moderated by other related medications and needed to stop the treatment. Persistence of these conditions caused repeated interruption of treatment and resulted in termination of treatment. Therefore, the long-term use of SMYAD is safer for patients with post-stroke epilepsy. Another severe adverse effect was suicide in LEV group as shown in Table 1. Although the comparison of suicide number between the groups showed in Table 1 had no statistical significance $(P>$ $0.05)$, there was no suicide case observed in SMYAD group. This may be due to the better effects of SMYAD on improving neurological functions.

Table 3. The Adverse Events of Treatment with LEV and SMYAD.

\begin{tabular}{llll}
\hline Group & LEV & SMYAD & $P$ Value \\
\hline Total Cases Enrolled (n) & 56 & 56 & \\
Cases Having Symptoms of Adverse & 28 & $6^{\mathrm{b}}$ & 0.000 \\
Events (n) & & $0^{\mathrm{b}}$ & 0.006 \\
Symptoms of Adverse Events: Rash (n) & 7 & $0^{\mathrm{b}}$ & 0.012 \\
Fatigue (n) & 6 & $0^{\mathrm{b}}$ & 0.012 \\
Somnolence (n) & 6 & $0^{\mathrm{b}}$ & 0.022 \\
Headache (n) & 5 & $0^{\mathrm{b}}$ & 0.042 \\
Dizziness (n) & 4 & $6^{\mathrm{b}}$ & 0.012 \\
Diarrhea (n) & 0 & & \\
\hline
\end{tabular}

Note: a: $P>0.05$, b: $P<0.05$ ( $\chi^{2}$ test, compared with LEV group).

\section{Discussion}

The above data show that SMYAD had comparable effect on controlling post-stroke epilepsy as LEV but was superior to LEV in quickening the time of epilepsy control, improving EEG patterns, decreasing NIHSS score and the relapse rate of epilepsy. Additionally, treatment using SMYAD caused fewer symptoms of adverse events than LEV. Published evidence shows that LEV has better therapeutic and neuroprotective effects on all forms of epilepsy with fewer adverse effects than other anti-epileptic drugs [27]. However, the price of LEV was relatively high for some patients. The daily cost of LEV was about $39 \%$ higher than the cost of SMYAD to our patients with post-stroke epilepsy. Long-term use of LEV was an economic burden to some patients and their families. This resulted in discontinuation of LEV treatment due to some patients switching to other low-cost anti-epileptic drugs or to SMYAD. Therefore, SMYAD was more accessible and acceptable to patients. As to the effects of SMYAD on improving EEG and reducing the relapse rate of epilepsy, it might be related to the role of SMYAD in decreasing iron content in the brain. Our recent studies found that SMYAD could remarkably reduce heme and iron levels in rat brain in animal ICH models [39]. However, more evidence will be needed to advance this idea.

According to the principles of TCM, the therapeutic methods for epilepsy include tranquillizing, cooling heat, dissolving phlegm, and removing blood clots by different factors that induce epilepsy. Post-stroke epilepsy results from either hemorrhagic or ischemic brain injury which are regarded as blood stasis in the brain, and blood stasis can further cause excessive heat in the tissue. Therefore, lowering heat and removing blood clots become important components of TCM therapy for post-stroke epilepsy. The herbs in SMYAD such as Jin-yin-hua (Honeysuckle flower), Xuan-shen (Scrophularia root), and Dang-gui (Chinese angelica root) are considered to have very important effects on cooling heat and removing blood stasis. It is therefore considered beneficial for treating post-stroke epilepsy [33].

Clinically, post-stroke seizures are classified as early seizures (within 14 days of stroke) and late seizures (after 14 days of stroke). Post-stroke epilepsy induced by late seizures was found to be more frequent than that induced by early seizures [1]. Early seizures may be caused by an abnormal discharge of local neurons due to the increased excitatory amino acids such as glutamic acid in the brain, and the late seizures are caused by gliosis and meningeal scar [1]. Experimental studies found that epilepsy after stroke was related to the increased iron ions or heme content in the brain, because direct injection of iron ions (such as in the form of ferric chloride) or heme into the brain of rats can cause seizures and long-term EEG abnormalities [13]. Iron ions mediate oxidation-reduction reactions in cell fluids and intercellular solutions, produce oxygen free radicals, cause oxidative damage to cell membrane and lead to release of glutamate and aspartic acid in cells. These changes in the brain could directly induce epilepsy and abnormal EEG. In addition, iron ions can also induce inflammatory reaction in brain tissue and increase the expression of inflammatory cytokines such as IL-1 $\beta$, IL-6, and TNF- $\alpha$ [18, 19]. The increased expression of inflammatory cytokines in the brain can also cause membrane damage and leakage of excitatory amino acid, leading to continuously firing neurons, seizure induction and abnormal EEG [20]. Therefore, increased iron in the brain is an important factor for epilepsy after stroke. Studies in animal models show accumulated residual iron in the brain tissue after cerebral hemorrhage [17]. As to ischemic stroke, peripheral mononuclear phagocytes rapidly enter the injured site, and then stay in the brain for a long time after stroke. These phagocytes carry large amounts of iron ions which add to the oxidative damage and chronic inflammation in the brain tissue. In addition, increased heme synthesis in brain cells to resist ischemic hypoxia at the early stage of cerebral ischemia, is also a resource of iron ions in the brain [14]. Therefore, iron plays an important role in post-stroke epilepsy, and eliminating excessive iron in the brain would be an important treatment for post-stroke epilepsy.

Currently all anti-epileptic drugs are used to stabilize the cell membrane and to inhibit the abnormal discharge of neurons in order to achieve the goal of controlling epilepsy. The mechanism of such treatment does not involve removing excessive iron ions in the brain. It is therefore difficult to improve the abnormal EEG or reduce the relapse rate of epilepsy after anti-epilepsy treatment. As mentioned above, according to the theory of TCM, SMYAD would have the effect of clearing heat and toxins, promoting blood circulation and removing blood stasis [30]. The SMYAD decoction has been found to inhibit the expression of 
inflammatory cytokines such as IL-1 $\beta$, IL-6, TNF- $\alpha$ [38], and decrease iron content in the brain in animal ICH models [39]. This may be the mechanism through which SMYAD inhibits epilepsy, improves abnormal EEG, and lowers NIHSS score as showed in the data presented here.

\section{Conclusion}

SMYAD was well-tolerated and showed beneficial effects for controlling post-stroke epilepsy through remission of seizures, reduction in the rate of relapse, and improved EEG patterns. Its beneficial effect was also shown by decreased NIHSS score. We conclude that SMYAD is potentially an effective remedy for patients with post-stroke epilepsy. However, large scale randomized controlled trials are needed to establish the efficacy and tolerability for wider acceptance of SMYAD.

\section{Author Contributions}

Drs Shisheng Jiang, Shuangteng He, Yuming Han, and Hongmei Wang accomplished the clinical observation for patients and collected the data. Hongmei Wang and Feizhou He assigned the medications and medicinal herbs to patients and verified the data. Drs Shuangteng He, Yuming Han, and Mukut Sharma analyzed the data and wrote the paper.

\section{Disclosure/Conflict of Interests}

All authors of this study declared that they had no conflict of interest.

The views expressed in this article are those of the authors and do not necessarily reflect the position or policy of the Department of Veterans Affairs or the United States government.

\section{Acknowledgements}

This study was supported by the resources from the Midwest Veterans' Biomedical Research Foundation (MVBRF), USA, partly supported from NIH/NIDDK R01DK107490 (to Mukut Sharma), a grant from the National Natural Scientific Foundation of China (No. 81373601 to Shisheng Jiang), and a key grant from the Bureau of Science and Technology, Hunan Province, China (No. 2012WK3008 to Shisheng Jiang).

\section{References}

[1] Camilo O, Goldstein LB. (2004) Seizures and epilepsy after ischemic stroke. Stroke. 35 (7): 1769-1775.

[2] Sykes L, Wood E, Kwan J. (2014) Antiepileptic drugs for the primary and secondary prevention of seizures after stroke. Cochrane Database Syst Rev. 1: CD005398.

[3] Jung S, Schindler K, Findling O, Mono ML, Fischer U, Gralla J, El-Koussy M, Weck A, Galimanis A, Brekenfeld C, Schroth G, Mattle HP, Arnold M. (2012) Adverse effect of early epileptic seizures in patients receiving endovascular therapy for acute stroke. Stroke. 43 (6): 1584-1590.

[4] Nicolo JP, O'Brien TJ, Kwan P. Role of cerebral glutamate in post-stroke epileptogenesis. Neuroimage Clin. 2019; 24: 102069.

[5] Consoli D, Bosco D, Postorino P, Galati F, Plastino M, Perticoni GF, Ottonello GA, Passarella B, Ricci S, Neri G, Toni D; EPIC Study. (2012) Levetiracetam versus carbamazepine in patients with late poststroke seizures: a multicenter prospective randomized open-label study (EpIC Project). Cerebrovasc Dis. 34 (4): 282-289.

[6] Szaflarski JP, Rackley AY, Kleindorfer DO, Khoury J, Woo D, Miller R, Alwell K, Broderick JP, Kissela BM. (2008) Incidence of seizures in the acute phase of stroke: a population-based study. Epilepsia. 49 (6): 974-981.

[7] Chen TC, Chen YY, Cheng PY, Lai CH. (2012) The incidence rate of post-stroke epilepsy: a 5-year follow-up study in Taiwan. Epilepsy Res. 102 (3): 188-194.

[8] Krakow K, Sitzer M, Rosenow F, Steinmetz H, Foerch C; Arbeitsgruppe Schlaganfall Hessen. (2010) Predictors of acute poststroke seizures. Cerebrovasc Dis. 30 (6): 584-589.

[9] D'Alessandro R, Tinuper P, Ferrara R, Cortelli P, Pazzaglia P, Sabattini L, Frank G, Lugaresi E. (1982) CT scan prediction of late post-traumatic epilepsy. J Neurol Neurosurg Psychiatry. 45 (12): 1153-1155.

[10] Faught E, Peters D, Bartolucci A, Moore L, Miller PC. (1989) Seizures after primary intracerebral hemorrhage. Neurology. 39 (8): 1089-1093.

[11] Willmore, L. J. and Ueda, Y. (2009) Posttraumatic epilepsy: hemorrhage, free radicals and the molecular regulation of glutamate. Neurochem Res. 34, 688-697.

[12] Willmore LJ, Sypert GW, Munson JV, Hurd RW. (1978) Chronic focal epileptiform discharges induced by injection of iron into rat and cat cortex. Science. 200 (4349): 1501-1503.

[13] Sharma V, Babu PP, Singh A, Singh S, Singh R. (2007) Iron-induced experimental cortical seizures: electroencephalographic mapping of seizure spread in the subcortical brain areas. Seizure. 16 (8): 680-690.

[14] Engström ER, Hillered L, Flink R, Kihlström L, Lindquist C, Nie JX, Olsson Y, Silander HC. (2001) Extracellular amino acid levels measured with intracerebral microdialysis in the model of posttraumatic epilepsy induced by intracortical iron injection. Epilepsy Res. 43 (2): 135-144.

[15] Park UJ1, Lee YA, Won SM, Lee JH, Kang SH, Springer JE, Lee YB, Gwag BJ. (2011) Blood-derived iron mediates free radical production and neuronal death in the hippocampal CA1 area following transient forebrain ischemia in rat. Acta Neuropathol. 121 (4): 459-473.

[16] Lipscomb DC1, Gorman LG, Traystman RJ, Hurn PD. (1998) Low molecular weight iron in cerebral ischemic acidosis in vivo. Stroke. 29 (2): 487-492.

[17] Wagener, F. A., Volk, H. D., Willis, D., Abraham, N. G., Soares, M. P., Adema, G. J., and Figdor, C. G. (2003) Different faces of the heme-heme oxygenase system in inflammation. Pharmacol Rev. 55, 551-571. 
[18] Figueiredo, R. T., Fernandez, P. L., Mourão-As, D. S., Porto, B N., Dutra, F. F., Alves, L. S., Oliveira, M. F., Oliveira, P. L., Graça-Souza, A. V., and Bozza, M. T. (2007) Characterization of heme as activator of Toll-like receptor 4. J Biol Chem. 282, 20221-20229.

[19] Lin, S., Yin, Q., Zhong, Q., Lv, F. L., Zhou, Y., Li, J. Q., Wang, J. Z., Su, B. Y., and Yang, Q. W. (2012) Heme activates TLR4-mediated inflammatory injury via MyD88/TRIF signaling pathway in intracerebral hemorrhage. J Neuroinflammation. 9, 46.

[20] Devi PU, Manocha A, Vohora D. (2008) Seizures, antiepileptics, antioxidants and oxidative stress: an insight for researchers. Expert Opin Pharmacother. 9 (18): 3169-3177.

[21] Vezzani A, Friedman A, Dingledine RJ. (2013) The role of inflammation in epileptogenesis. Neuropharmacology. 69: 16-24.

[22] Naidech AM, Garg RK, Liebling S, Levasseur K, Macken MP, Schuele SU, Batjer HH. (2009) Anticonvulsant use and outcomes after intracerebral hemorrhage. Stroke. 40 (12): $3810-3815$.

[23] Messé SR, Sansing LH, Cucchiara BL, Herman ST, Lyden PD, Kasner SE; CHANT investigators. (2009) Prophylactic antiepileptic drug use is associated with poor outcome following ICH. Neurocrit Care. 11 (1): 38-44.

[24] Zubkov AY1, Wijdicks EF. (2008) Antiepileptic drugs in aneurysmal subarachnoid hemorrhage. Rev Neurol Dis. 5 (4): 178-181.

[25] Szaflarski JP, Sangha KS, Lindsell CJ, Shutter LA. (2010) Prospective, randomized, single-blinded comparative trial of intravenous levetiracetam versus phenytoin for seizure prophylaxis. Neurocrit Care. 12 (2): 165-172.

[26] Myint PK, Staufenberg EFA, Sabanathan K. (2006) Post-stroke seizure and post-stroke epilepsy. Postgrad. Med. J. 82 568-572.

[27] Shetty AK. (2013) Prospects of levetiracetam as a neuroprotective drug against status epilepticus, traumatic brain injury, and stroke. Front Neurol. 4: 172.

[28] Woo KM, Yang SY, Cho KT. (2012) Seizures after spontaneous intracerebral hemorrhage. J Korean Neurosurg Soc. 52 (4): 312-319.

[29] Selim M, Yeatts S, Goldstein JN, Gomes J, Greenberg S, Morgenstern LB, Schlaug G, Torbey M, Waldman B, Xi G, Palesch Y, Deferoxamine Mesylate in Intracerebral Hemorrhage Investigators. (2011) Safety and tolerability of deferoxamine mesylate in patients with acute intracerebral hemorrhage. Stroke. 42 (11): 3067-3074.

[30] He S, Jiang S, and He F. (2001) Successful treatment of cerebral abscess with the Decoction of Si Miao Yong An Tang (in Chinese), Chinese Journal of Traditional Chinese Medicine. 41 (12): 552-553.

[31] iang S, He S, Han Y, Xia A, Wang H, Wu C, He F. (2011) The Decoction of Si Miao Yong An on the acute phase of intracerebral hemorrhage. Chinese J. Basic Medicine for Traditional Chinese Medicine. 17 (4): 314-318.

[32] Jiang S, He S, Han Y, Xia A, Wang H, He F. (2011) The effects of Simiao Yongan Decoction on preventing recurrent stroke. J. Emergency of Traditional Chinese Medicine. 20 (11): 1721-1723.
[33] Jiang S, Han Y, Xia A, Wang H, He F, He S. (2016) The effects of Si-Miao-Yong-An decoction in treatment of post-stroke epilepsy: a report of 35 cases. Hunan Journal of Traditional Chinese Medicine. 32 (2): 1-4.

[34] Su C, Wang Q, Zhang H, Jiao W, Luo H, Li L, Chen X, Liu B, Yu X, Li S, Wang W, Guo S. (2019) Si-Miao-Yong-An Decoction Protects Against Cardiac Hypertrophy and Dysfunction by Inhibiting Platelet Aggregation and Activation. Front Pharmacol. 18 (10): 990.

[35] Zhao Y, Jiang Y, Chen Y, Zhang F, Zhang X, Zhu L, Yao X. (2020) Dissection of mechanisms of Chinese medicinal formula Si-Miao-Yong-an decoction protects against cardiac hypertrophy and fibrosis in isoprenaline-induced heart failure. J Ethnopharmacol. 248: 112050.

[36] Ren Y, Chen X, Li P, Zhang H, Su C, Zeng Z, Wu Y, Xie X, Wang Q, Han J, Guo S, Liu B, Wang W. (2019) Si-Miao-Yong-An decoction ameliorates cardiac function through restoring the equilibrium of SOD and NOX2 in heart failure mice. Pharmacol Res. 146: 104318.

[37] Zhang JP, Xu YZ, Li M, Li LJ, Peng L, Zhang GY, Yang C, Zhou YN, 2010. Effect of Simiao Yong'an decoction on oxidative stress and inflammation on atherosclerosis model rabbits. Journal of Traditional Chinese Medicine 51: 72-74. (in Chinese).

[38] Peng L, Li M, Xu YZ, Zhang GY, Yang C, Zhou YN, Li LJ, Zhang JP. (2012). Effect of Si-Miao-Yong-An on the stability of atherosclerotic plaque in a diet-induced rabbit model. J. Ethnopharmacol. 143 (1): 241-248.

[39] Jiang S, Han Y, Wang H, He F, He S. The effects and mechanisms of Si-Miao-Yong-An decoction on decreasing hemin and iron contents in rat brain after intracerebral hemorrhage. Chinese Journal of Traditional Chinese Medicine Pharmacology. 2017; 32 (3): 1044-1049.

[40] Broderick J, Connolly S, Feldmann E, Hanley D, Kase C, Krieger D, Mayberg M, Morgenstern L, Ogilvy CS, Vespa P, Zuccarello M. Guidelines for the management of spontaneous intracerebral hemorrhage in adults: 2007 update: a guideline from the American Heart Association/American Stroke Association Stroke Council, High Blood Pressure Research Council, and the Quality of Care and Outcomes in Research Interdisciplinary Working Group. Stroke. 2007; 38 (6): 2001-2023.

[41] Sacco RL, Adams R, Albers G, Alberts MJ, Benavente O, Furie K, Goldstein LB, Gorelick P, Halperin J, Harbaugh R, Johnston SC, Katzan I, Kelly-Hayes M, Kenton EJ, Marks M, Schwamm LH, Tomsick T; American Heart Association/American Stroke Association Council on Stroke; Council on Cardiovascular Radiology and Intervention; American Academy of Neurology. (2006) Guidelines for prevention of stroke in patients with ischemic stroke or transient ischemic attack: a statement for healthcare professionals from the American Heart Association/American Stroke Association Council on Stroke: co-sponsored by the Council on Cardiovascular Radiology and Intervention: the American Academy of Neurology affirms the value of this guideline. Circulation. 113 (10): e409-449.

[42] Berg AT, Berkovic SF, Brodie MJ, Buchhalter J, Cross JH, van Emde Boas W, Engel J, French J, Glauser TA, Mathern GW, Moshé SL, Nordli D, Plouin P, Scheffer IE. (2010) Revised terminology and concepts for organization of seizures and epilepsies: report of the ILAE Commission on Classification and Terminology, 2005-2009. Epilepsia. 51 (4): 676-685. 